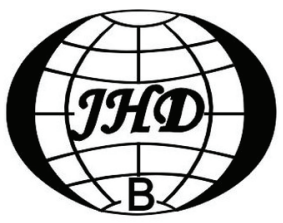

sdlj.chinajournal.net.cn

\title{
LARGE-EDDY SIMULATION OF THREE-DIMENSIONAL TURBULENT FLOW AROUND A CIRCULAR PIER*
}

\author{
ZHAO Wei, Huhe Aode \\ Institute of Mechanics, Chinese Academy of Sciences, Beijing 100080, China, E-mail:zhaoweihsei@sina.com.cn
}

(Received April 7, 2006)

\begin{abstract}
In this article, the turbulent flow field and the scouring mechanism around a circular pier were numerically investigated using the Large Eddy Simulation (LES) method for three scouring holes. The effects of the bottom topographies on the flow structure were studied in detail. The results show that at the downstream of the pier, as the scouring depth increases, the bed shear stress decreases and approaches to the undisturbed shear stress, however, the turbulent intensity, the fluctuating pressure, and the vertical pressure gradient increase gradually.
\end{abstract}

KEY WORDS: scouring mechanism, Large Eddy Simulation (LES), bed shear stress, fluctuating pressure, turbulent intensity

\section{INTRODUCTION}

The local scouring around a circular pier occurs rather frequently in engineering, which is closely related to the flow field and the movable bed. The flow field is a complex three-dimensional unsteady flow. The complexity is further magnified due to the interaction between the flow and the movable bed during scouring. Hitherto, there have been a number of investigations on the problem, but most of the studies focused on the formation of the maximum scouring depth based on the small-scale laboratory experiments and a few field studies. Therefore, the resultant formulae are not always reasonable under practical conditions, and only few studies have been concerned with the scouring mechanism. Dargahi ${ }^{[1,2]}$ performed the detailed experimental investigations on the flow patterns and the velocity fields around a cylinder. $\mathrm{Li}^{[3]}$ and $\mathrm{Zhao}^{[4]}$ conducted some experiments to study the effects of the diameter of a cylinder and the approaching velocity on the scouring depth. Systematic experimental investigations are very expensive and time-consuming. Therefore, the numerical research has become more feasible due to the recent advances in computer applications.

In recent years, several numerical studies on the flow field and the bed variation around a circular pier have been carried out, which were mostly based on the Reynolds-Averaged Navier-Stokes (RANS) equations with different turbulence models ${ }^{[5-9]}$ Olsen and Malaaen ${ }^{[5]}$ neglected the transient terms in the RANS equations with the $k-\varepsilon$ turbulence model to simulate the flow field and give the bed shear stress. Kamil $^{[7]}$ calculated the flow field around a circular cylinder in the scouring holes of different sizes. Salaheldin ${ }^{[8]}$ simulated the flow field around circular piers with different turbulence models.

Under practical conditions, turbulence plays an important role in the scouring processes. The effect of turbulence must be reasonably accounted for in numerical models. Rodi ${ }^{[10]}$ compared the results of the LES approach and RANS equations for the flow around the bluff bodies with those of experimental measurements. It is shown that in all RANS calculations with various versions of turbulence models, the turbulent fluctuations were considerably underpredicted, especially in the case that the large-scale eddy structures or unsteady Vortex-shedding dominate the turbulent transport process. The LES approach is more suitable for this complex flow and might give better simulation.

The objective of the present study is to investigate the turbulent flow field and the scouring mechanism around a circular pier during scouring, especially at the downstream of the pier. The investigation is carried out in the framework of

\footnotetext{
* Biography: ZHAO Wei(1973-),Male,Ph. D.
} 
transient and averaging approaches. The effects of energy-containing turbulent eddies, the turbulent intensities, and the fluctuating pressures on the local scouring are revealed in detail with the development of the scouring hole. The flow field is simulated with the LES approach, and the simulated results are compared with the experimental data ${ }^{[11]}$. The approaching Reynolds number is $\operatorname{Re}(D)=7040$, where $D$ is the pier diameter).

\section{MATHEMATICAL MODEL}

\subsection{Governing Equations}

The LES approach is used in this article. In the LES approach, the scales larger than the filter width are resolved with the spatially filtered 3-D, time-dependent Navier-Stokes equations

$$
\begin{aligned}
& \frac{\partial \bar{u}_{i}}{\partial x_{i}}=0 \\
& \frac{\partial}{\partial t}\left(\rho \bar{u}_{i}\right)+\frac{\partial}{\partial x_{j}}\left(\rho \bar{u}_{i} \bar{u}_{j}\right)=\frac{\partial}{\partial x_{j}}\left(\mu \frac{\partial \bar{u}_{i}}{\partial x_{j}}\right)-\frac{\partial \bar{p}}{\partial x_{i}}-\frac{\partial \tau_{i j}}{\partial x_{j}}
\end{aligned}
$$

where $\rho$ and $\mu$ is density and viscosity, respectively, and $\tau_{i j}$ is the subgrid-scale stress, given as

$$
\tau_{i j}=\rho \overline{u_{i} u_{j}}-\rho \bar{u}_{i} \bar{u}_{j}
$$

The effect of the unresolved small-scale motion is simulated with the subgrid-scale model. In the present simulations, the Smagorinsky-Lilly eddy viscosity model has been applied, wherein

$$
\begin{aligned}
& \tau_{i j}-\frac{1}{3} \tau_{k k} \delta_{i j}=-2 \mu_{t} \bar{S}_{i j} \\
& \mu_{t}=\rho L_{s}^{2} \sqrt{2 \bar{S}_{i j} \bar{S}_{i j}} \\
& \bar{S}_{i j}=\frac{1}{2}\left(\frac{\partial \bar{u}_{i}}{\partial x_{j}}+\frac{\partial \bar{u}_{j}}{\partial x_{i}}\right) \\
& L_{s}=\min \left(\kappa d, C_{s} V^{1 / 3}\right)
\end{aligned}
$$

where $\mu_{t}$ is the subgrid-scale turbulent viscosity, $\kappa=0.42, C_{s}=0.1, d$ is the distance to the closest wall, and $V$ is the volume of the computational cell.

The governing equations are discretized using a second-order accurate cell-centered finite-volume method with the upwinding scheme. A second-order implicit scheme is used for all time-dependent terms, which is unconditionally stable with respect to the time-step size. The pressure-velocity coupling scheme of the Pressure-Implicit with Splitting of Operators (PISO) is used, which can obviously reduce the number of iterations required for convergence.

\subsection{Calculation domain and boundary conditions}

The turbulent flow fields around a circular pier for a flat bed and two scouring holes are simulated. The scouring bed forms are obtained at two intermediate instants in a scouring process of the laboratory experiment ${ }^{[11]}$. The first scouring hole with the maximum scouring depth of $S=0.375 D$ branches from two sides of the pier, which is not washed out in the symmetry plane downstream of the pier. The second maximum scouring depth is $S=0.85 \mathrm{D}$, and the scouring form is approximately a truncated cone. The calculation domain is $30 D \times 12 D \times 3 D$ (Fig.1). To investigate the flow field near bed in detail, the unstructured grids are used and the near-wall mesh spacing is $Z^{+}<5$. The total grid number is 1390820 (see Fig.2).

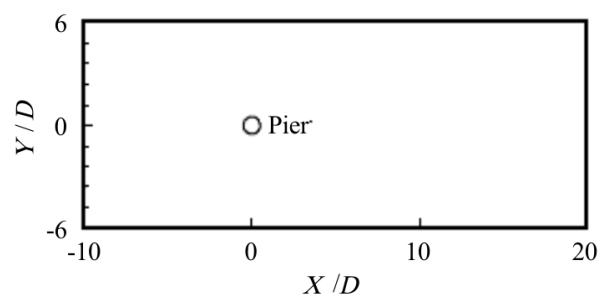

Fig.1 Calculation domain

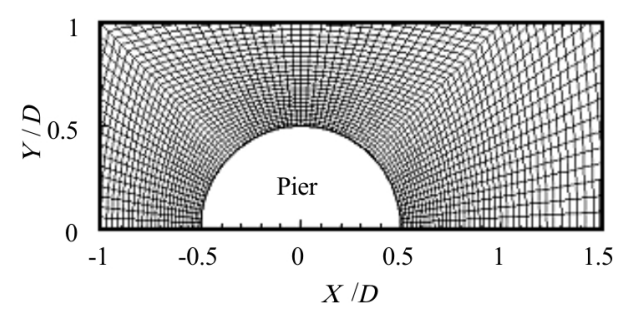

Fig.2 Grid near the pier (local)

At the inlet boundary $(X=-10 D)$, the transverse velocity is set to zero, and the longitudinal velocity is composed of the mean and fluctuating velocities, a vanishing gradient of velocity is applied at the outlet boundary $(X=20 D)$, a symmetry boundary condition is prescribed at the top flat surface boundary and the side boundaries $(Y= \pm 6 D)$, at the bed and pier surface, the non-slip and no-penetration boundary conditions are specified. 


\section{RESULTS AND DISCUSSION}

\subsection{Flow patterns}

To investigate the local scouring mechanism around a circular pier, the three-dimensional turbulent flow fields near the bed were accurately simulated. The transient flow patterns in the vertical plane and in the horizontal plane near bed $(0.02 D$ above the bed) are, respectively, shown in Fig.3.
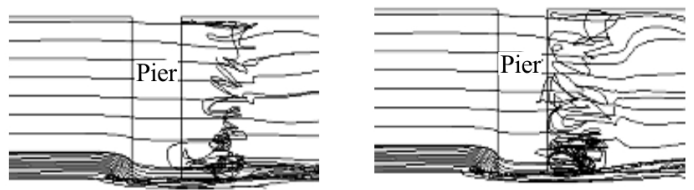

$S / D=0$, vertical plane

(a) $T=0$

(b) $T=\pi / 4$
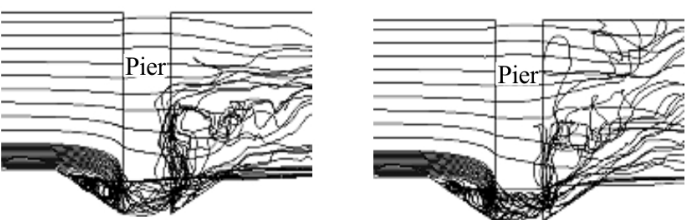

$S / D=0.85$, vertical plane

(c) $T=0$

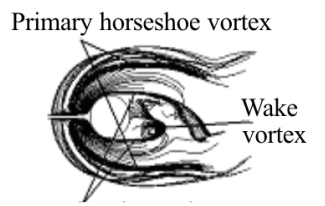

Secondary horseshoe vortex (d) $T=\pi / 4$

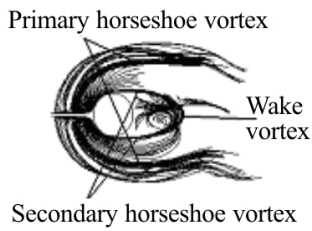

Secondary horseshoe vortex

$$
\text { (f) } T=\pi / 4
$$
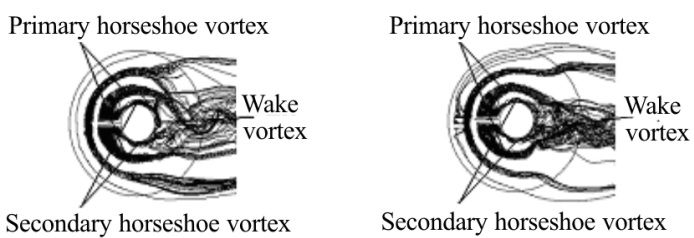

$S / D=0.85$, horizontal plane

(g) $T=0$

(h) $T=\pi / 4$

Fig.3 The instantaneous flow patterns around a circular pier over a period

Figures 3(a) to 3(d) show the particles motions in the vertical plane of the pier. For a flat bed, the bed particles are lifted up only in the area of $X=1 D-2 D$ (see Figs. 3(a) and 3(b)). With the evolution of the scouring hole, the wake vortex shedding is more complicated. When the scouring depth approaches to $S=0.375 D$, one part of the rising particles drops back to the bed and another part is carried downstream of the pier. After the scouring hole has been washed out in the symmetry plane downstream of the pier, one part of the particles from the near-bed region is sucked into the main flow and suspended and another part is carried downstream along the bed surface (see Figs.3(c) and 3(d)). The strength of the suction changes with the wake vortex shedding.

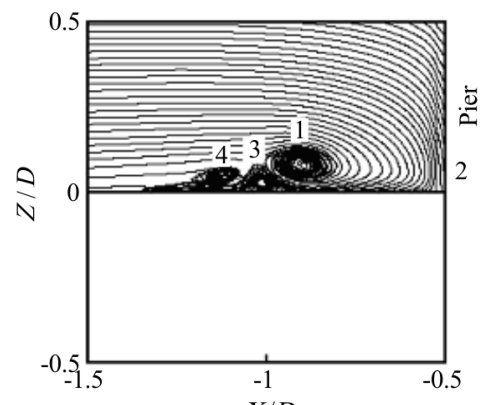

(a) $\underset{S}{X / D} / D$

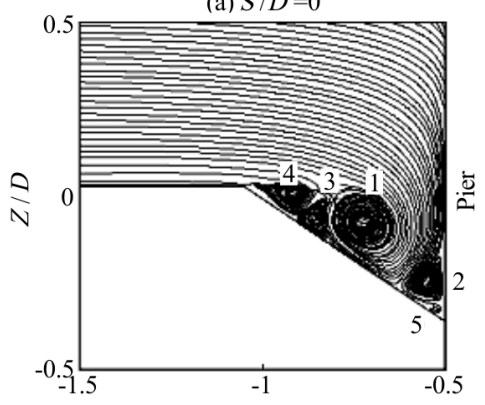

(b) $S / D=0.375$

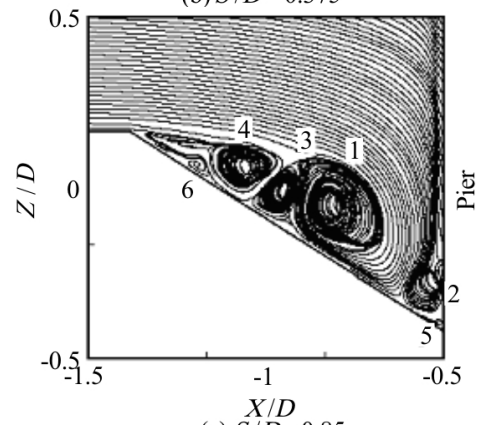

(c) $S / D=0.85$

Fig.4 Instantaneous flow patterns of the horse vortex system

The instantaneous flow patterns upstream are described in detail in Fig. 4. The horseshoe vortex system contains a primary horseshoe vortex (Vortex-1), a horseshoe vortex close to foundation (Vortex-2), and the secondary horseshoe vortices (Vortex-3 V Vortex-6). The scale and the number of the horseshoe vortices increase with increasing scouring depth. The strength of the Vortex-1 first increases and then decreases at a certain instant during the scouring, and the maximum among these three cases is at $S=0.375 D$. The result is similar to that given by Yuhi et al. ${ }^{[12]}$, which has been obtained for $R e=2000$ with a DNS approach.

The downflow behaves like a vertical jet in drilling the bed and supplies fluid to the horseshoe 
vortices, especially to the Vortex-2. The strength of the downflow for these three cases is shown in Fig.5. The downward velocity increases with increasing scouring depth. For the scouring depth of $S / D=0.85$, the maximum velocity reaches $0.87 U$, where $U$ is the approaching velocity.

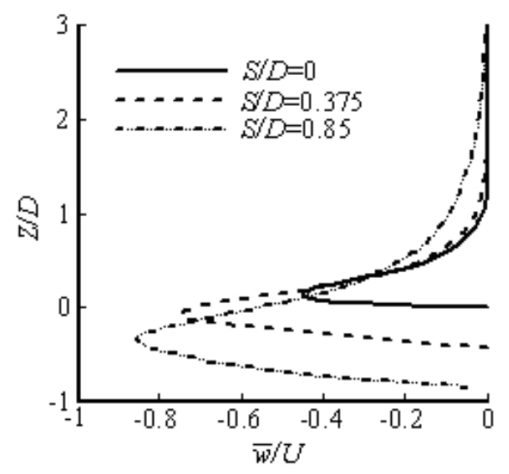

Fig.5 Downflow velocity in front of the pier

Figures 3(e)-3(h) show the flow patterns in the horizontal plane near the bed, from which the phenomena of the interaction between the horseshoe vortices and the wake vortices during the erosion can be seen. For a flat bed, the Vortex-2 moves along the pier surface and integrates with the wake vortices at the downstream of the pier. For the scouring depth of $S=0.375 D$, the Vortex-2 is influenced by the scouring bed and interacts more obviously with the wake vortices near the bed. As the scouring depth approaches to $S=0.85 D$, the horseshoe vortices are close to the pier face at the downstream of the pier. The interaction between the horseshoe vortices and the wake vortices are amplified.

Figure 6 shows the instantaneous vorticity components in the symmetry plane downstream of the pier, where $\omega_{x}, \omega_{y}, \omega_{z}$ are the streamwise vortivity, crosswise vorticity and vertical vorticity, respectively. For a flat bed, the vortices are vertically compressed and $\omega_{x}$ and $\omega_{y}$ increase near the bed. For the scouring depth of $S=0.85 D$, the wake vortices are confined within the souring hole, and $\omega_{x}, \omega_{y}, \omega_{z}$ are approximately of the same order of magnitude, which are twice larger than that for a flat bed.

\subsection{Mean velocity fields}

The mean velocity fields in the symmetry plane of the pier are shown in Fig. 7. At the upstream of pier, the flow patterns reflect the action of the horseshoe vortices and the downflow. In the wake region, the flow fields are much complex during the scouring. For a flat bed, three flow regions (see Fig. 7(a)), i.e., the reversal flow region, the streamwise flow region, and the transition region, have been observed. For the scouring depth of $S=0.375 \mathrm{D}$, the velocity filed is complex and the reversal flow region is longer than that for the flat bed. As the scouring depth approaches to $S=0.85 D$ (Fig. 7(b)), a strong upward flow near the pier and a powerful streamwise flow along the scouring bed can be clearly observed.

For the case of the flat bed, the mean velocity components $(\bar{u} / U, \bar{w} / U)$ and the root-mean-square fluctuating velocity components

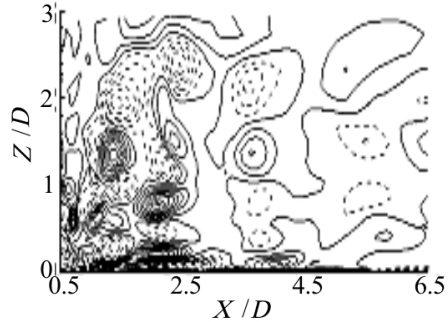

(a) $\omega_{x} D / U=-5 \sim 5, \quad S / D=0$

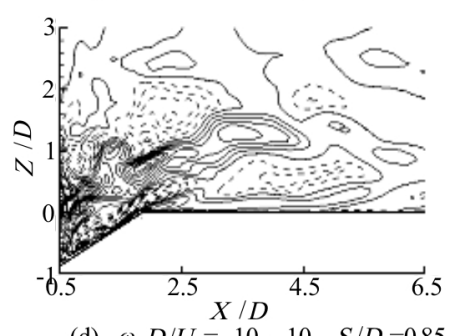

(d) $\omega, D / U=-10 \sim 10, \quad S / D=0.85$

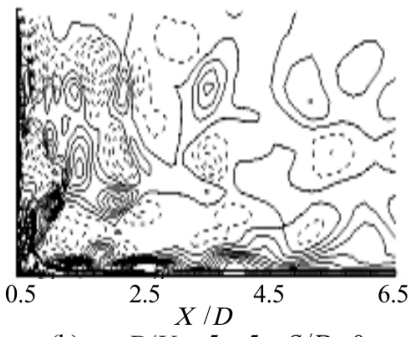

(b) $\omega_{y} D / U=-5 \sim 5, \quad S / D=0$

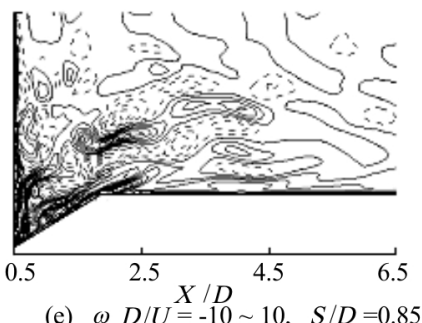

(e) $\omega_{v} D / U \stackrel{X}{=}-10 \sim 10, \quad S / D=0.85$

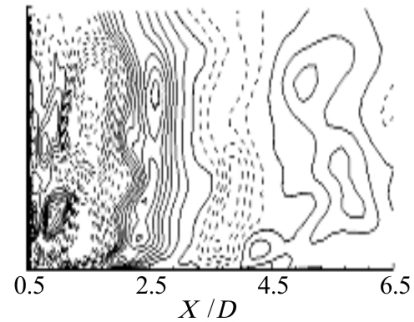

(c) $\omega_{z} D / U=-5 \sim 5, \quad S / D=0$

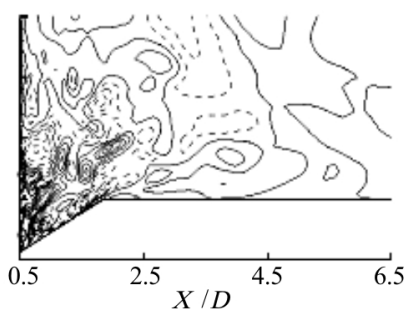

(f) $\omega_{z} D / U=-10 \sim 10, \quad S / D=0.85$

Fig.6 Contours of vorticity components in the symmetrical plane downstream of pier (Solid lines represent positive value, and dashed lines represent negative values) 


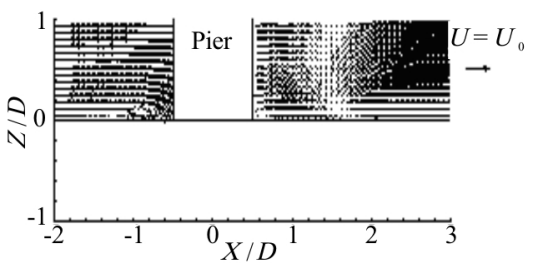

(a) $S / D=0$

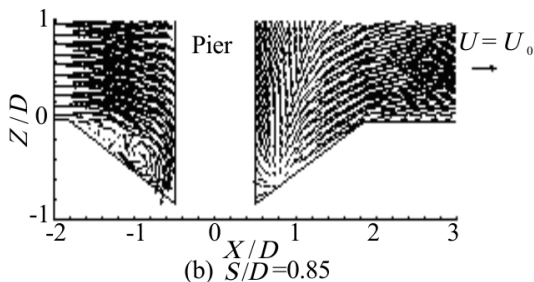

(b) $S / D=0.85$

Fig.7 Mean velocity field in the symmetrical plane of the pier

$\left(\sqrt{u^{\prime} u^{\prime}} / U, \sqrt{w^{\prime} w^{\prime}} / U\right)$ downstream of the pier are compared with the experimental data ${ }^{[11]}$ in Fig.8. It can be seen that the mean velocities are in good agreement with the measured values. The numerical results of the root-mean-square fluctuating velocity deviate from the experimental results because the incoming turbulence intensity in experiments is higher than that in calculations.

\subsection{Bed shear stresses}

The mean bed shear stresses for the three cases of scouring (normalized with respect to the undisturbed value $\tau_{0}$ ) are shown in Fig. 9. For the flat bed, the maximum bed shear stress appears at the pier shoulder of $54^{\circ}$ to the direction of the approaching flow. With the development of scouring, the position of the maximum moves downstream along the pier edge. When the scouring depth approaches to $S / D=0.85$, the large bed shear stresses have been observed at the rim of the scouring hole. Yuhi ${ }^{[12]}$ found the same tendency of the bed shear stresses during the scouring.

At the upstream, the amplification of the bed shear stress is directly influenced by the horseshoe vortices, and the shear stress magnitude is related to the strength of the horseshoe vortices (see Fig. 10(a)). It can also be seen from Fig. 10(a) that the magnitude of the bed shear stress increases as the scouring depth increases at first, the maximum appears at a certain scouring depth, and then decreases with the increase of the scouring depth. In the present case, the maximum appears at $S / D=0.375$.

The bed shear stresses downstream of the pier are shown in Fig. 10(b). With the development of scouring, the bed shear stress decreases and igradually becomes close to the value of the approaching shear stress. For the case of the flat bed, the maximum shear stress appears at $X=1.25 D$ and the value is $1.55 \tau_{0}$. For the scouring depth of $S / D=0.375$, the maximum shear stress occurs close to $\tau_{0}$. For the case of $S / D=0.85$, the bed shear stress has two peaks: one is near the pier foundation and the other is at the rim of the scouring hole.

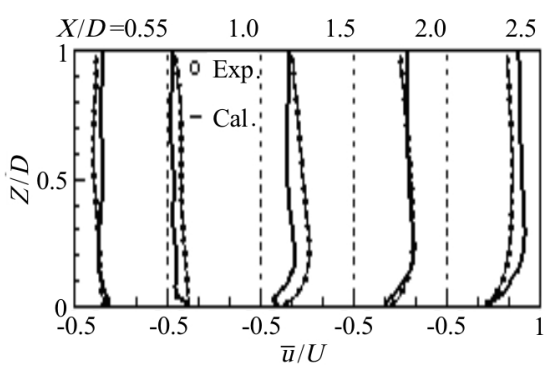

(a) $\bar{u} / U$

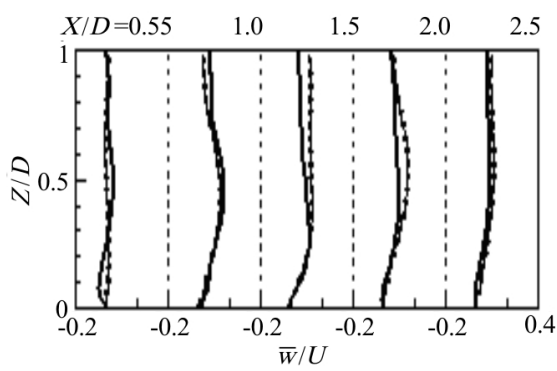

(b) $\bar{W} / U$

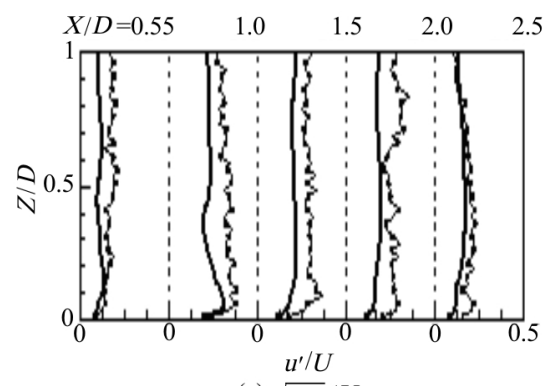

(c) $\sqrt{u^{\prime} u^{\prime}} / U$

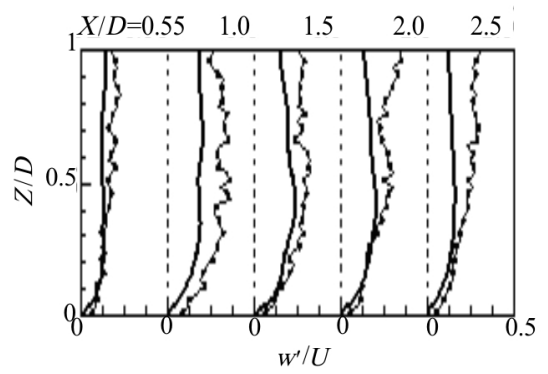

(d) $\sqrt{W^{\prime} W^{\prime}} / U$

Fig.8 Comparison of the mean velocity and the fluctuating velocity 


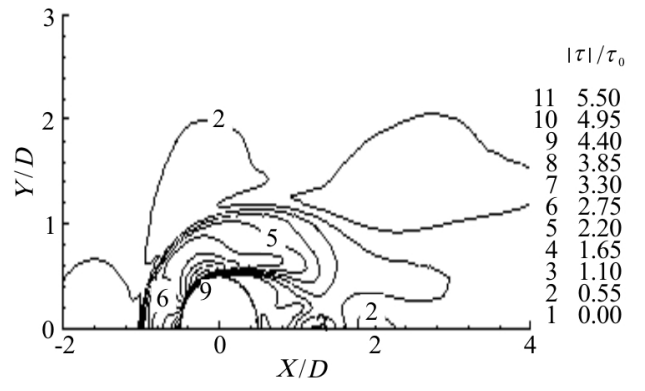

(a) $S / D=0$

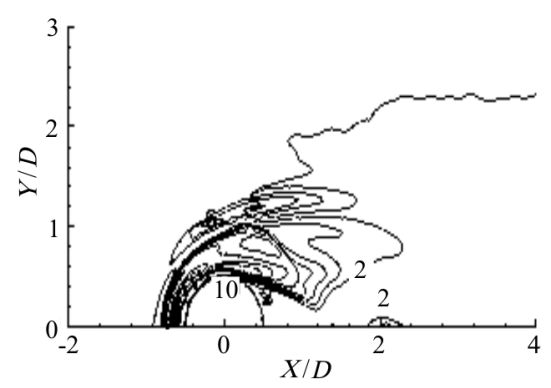

(b) $S / D=0.375$

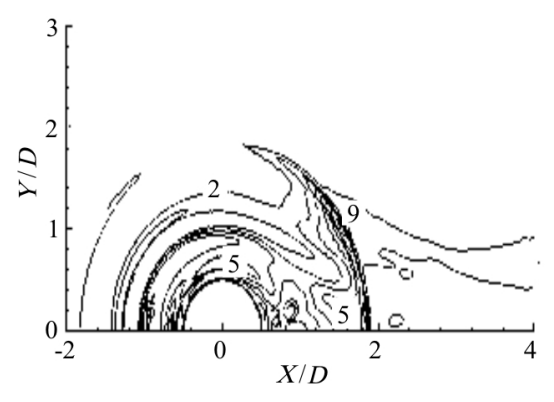

(c) $S / D=0.85$

Fig.9 Mean bed shear stresses around the pier

Up to now, the mean bed shear stress is still the basic parameter for establishing the model of sediment transport. But the sediment entrainment is a transient event, and the instantaneous bed shear stress may have the direct impact on the sediment motion. The transient bed shear stress varies obviously, especially in the wake region (see Fig. 11). For the flat bed, the maximum instantaneous bed shear stress reaches 1.5 times of the mean bed shear stress (see Figs.9(a) and 11(a)), and the location of the maximum transient value oscillates during the vortex shedding process. The ratio of the maximum instantaneous bed shear stress to the mean bed shear stress increases with the development of scouring. For the case of $S / D=0.375$, it is 2.5 (see Fig 9(b) and 11(b)), and as the scouring depth approaches to $S / D=0.85$, it rises to 3 (see Figs.9(c) and 11(c)).

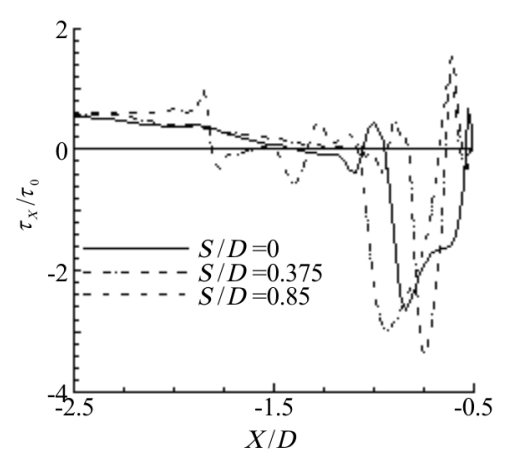

(a)upstream of the pier

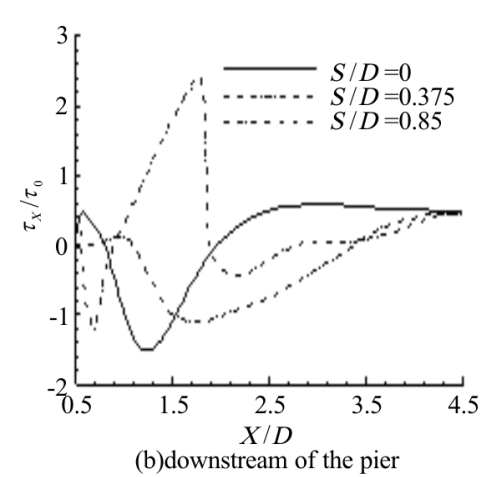

Fig.10 Mean bed shear stresses along the symmetrical line of the pier

\subsection{Turbulent kinetic energy}

The turbulence is a very important factor for the sediment motion, which is closely related to the complex flow field around the pier. Figure 12 shows the turbulent kinetic energy in the symmetry plane of the pier. For a flat bed, the turbulent intensity is large in the transition region (see Fig. 12(a)). For the scouring depth of $S / D=0.85$, the turbulent intensity considerably increases in the scouring hole, especially near the pier foundation (see Fig. 12(b)).

3.5 Pressure field

The mean and fluctuating pressure coefficients $\left(C_{P}=\left(P-P_{0}\right) /\left(2 \rho U^{2}\right)\right)$ in the symmetry plane of the pier are shown in Fig. 13. At the upstream of the pier, a low-pressure region exists near the center of the primary horseshoe vortex where the fluctuating pressure is also high. The pressure at the vortex center decreases as the scouring depth increases. In the near-wake region, the pressure becomes negative and the fluctuating pressure is higher than that at the upstream of the pier. For a flat bed, the negative pressure region and the large fluctuating pressure appear in the transition region. The maximum 


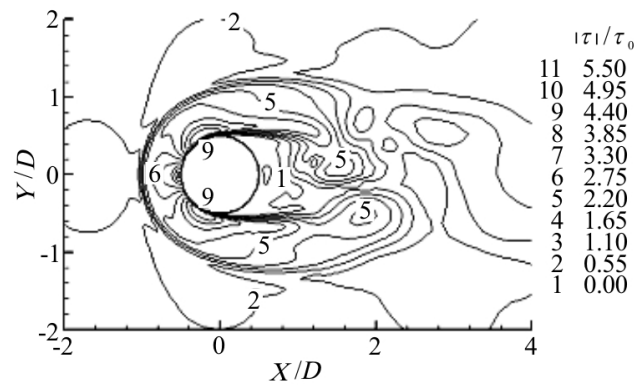

(a) $S / D=0$

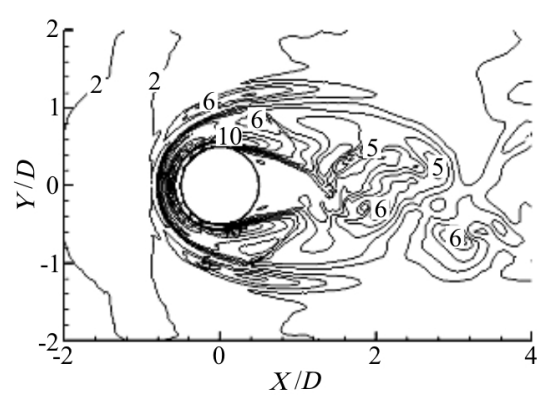

(b) $S / D=0.375$

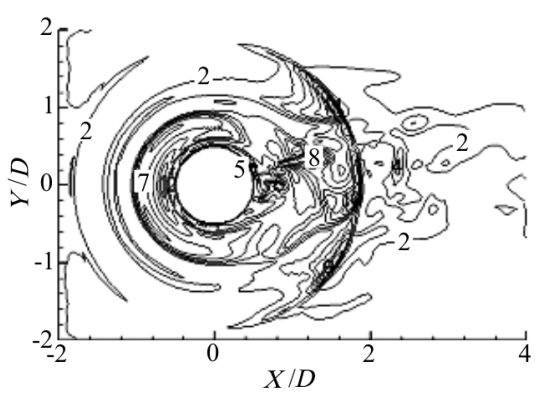

(c) $S / D=0.85$

Fig.11 Instantaneous bed shear stress around the circular pier

fluctuating pressure coefficient is 0.27 times the approaching pressure coefficient (see Fig. 13(b)). As the scouring depth approaches to $S / D=0.85$, there are two negative pressure regions that are located near the pier face and at the rim of the scouring hole, respectively. The maximum fluctuating pressure coefficient appears near the pier foundation, which is 0.5 times the approaching pressure coefficient (see Fig. 13(d)). The vertical pressure gradient is large in the scouring hole besides the streamwise pressure gradient. So the bed sediments near the foundation may be sucked up or carried downstream along the bed surface.

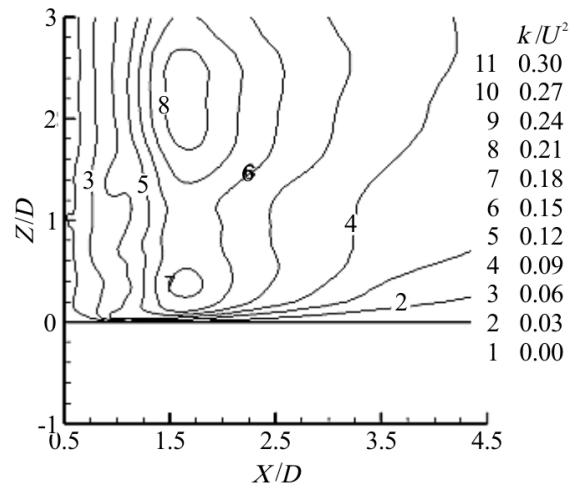

(a) $S / D=0$

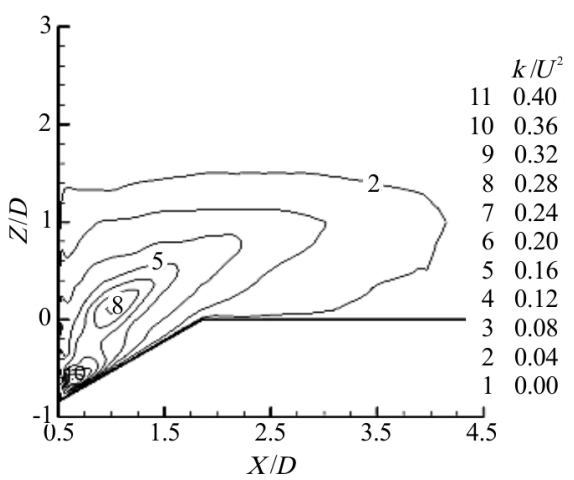

(b) $S / D=0$

Fig.12 Mean turbulent kinetic energy in the symmetry plane downstream of pier

\section{CONCLUSIONS}

The turbulent flow field and the scouring mechanism have been numerically investigated using the LES approach in this study. The main results are as follows.

In the traditional sediment transport model, the mean bed shear stress is a basic dynamic parameter. However, for the complex flow around a pier, especially at the downstream of the pier, the vorticity, the turbulent intensity, the fluctuating pressure, and the vertical negative pressure gradient exhibit considerable effects on the sediment motion.

The magnitude of the mean bed shear stress at the upstream of the pier is primarily influenced by the horseshoe vortices. At the downstream of the pier, the bed shear stress decreases gradually and approaches to the undisturbed shear stress as the scouring depth increases. However, the maximum instantaneous bed shear stress is several times larger than the mean bed shear stress, which directly impact on the sediment transport. 


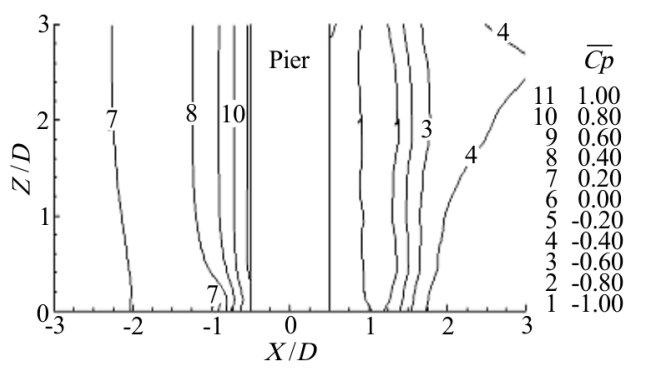

(a) $S / D=0$,mean pressure

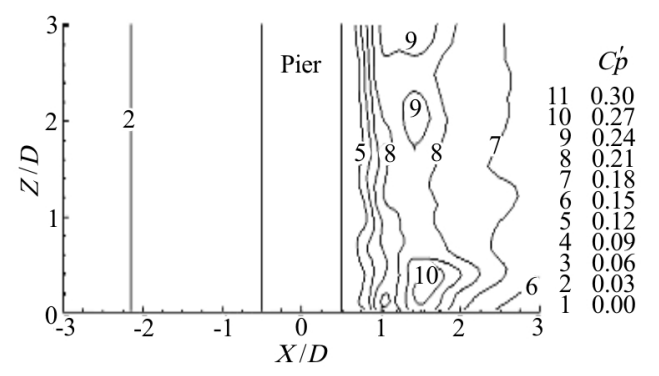

(b) $S / D=0$,fluctuating pressure

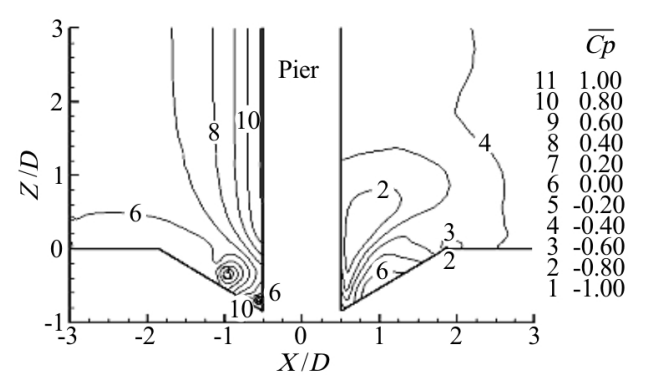

(c) $S / D=0.85$, mean pressure

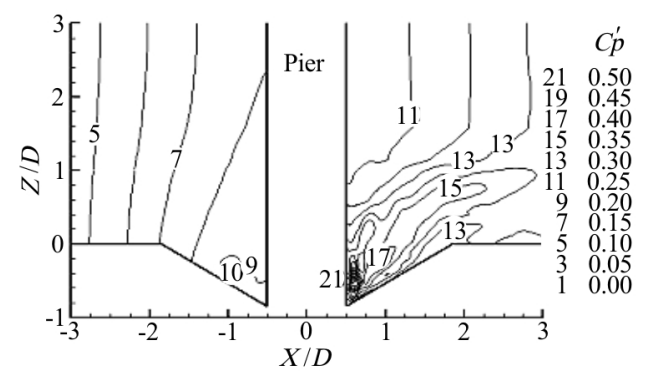

(d) $S / D=0.85$,fluctuating pressure

Fig.13 Mean and fluctuating pressure distributions in the symmetry plane of the pier

For the complex flow field around the pier, the turbulence plays an important role in the scouring process. As the scouring depth increases, the turbulent intensity increases. The maximum is one time larger for the scouring depth of $S / D=0.85$ than for flat bed.
The position of the maximum turbulence intensity gradually approaches to the pier face.

At the upstream of the pier, a low-pressure region exists near the center of the primary horseshoe vortex where the fluctuating pressure is also large. At the downstream of the pier, the pressure becomes negative and the fluctuating pressure is higher than that at the upstream of the pier. As the scouring depth increases, the maximum fluctuating pressure and the vertical pressure gradient gradually increase, which cause the bed sediments near the foundation to be sucked up.

\section{REFERENCES}

[1] DARGAHI B. The turbulent flow field around a circular cylinder[J]. Experiment in Fluids, 1989, 8(1-2): 1-12.

[2] DARGAHI B. Controlling mechanism of local scouring $[\mathrm{J}]$. ASCE Journal of Hydraulic Engineering, 1990, 116(10): 1197-1214.

[3] LI Lin-pu, CUI Li. Prediction of maximum scour depth around large-diameter cyliner under the effects of both wave and current[J]. Journal of Hydrodynamics, Ser. B, 2005, 17(1): 73-79.

[4] ZHAO Ming, TENG Bin, LI Lin-pu. Local scour around a large-scale vertical circular cylinder due to combined wave-current action[J]. Journal of Hydrodynamics, Ser. B, 2005, 17(4): 344-351.

[5] OLSEN N. R. B., MELAAEN M. C. Three-dimensional calculation of scour around cylinders[J]. ASCE Journal of Hydraulic Engineering, 1993, 119(9): 1048-1504.

[6] OLSEN N. R. B., KJELLESVIG H. M. Three-dimensional numerical flow modeling for estimation of maximum local scour depth[J]. Journal of Hydraulic Research, 1998, 36(4): 579-589.

[7] KAMIL H. M. Ali. Simulation of flow around piers[J]. Journal of Hydraulic Research, 2002, 40(2): 161-174.

[8] SALAHELDIN T. M., IMRAN J., CHAUDHRY M. H. Numerical modeling of three-dimensional flow field around circular piers[J]. ASCE Journal of Hydraulic Engineering, 2004, 130(2): 91-100.

[9] XUE Lei-ping, LIU Hua, LIU H. J. Three-dimensional numerical model for turbulent flow around a bottom-mounded pile[J]. Acta Mechanica Sinica, 2004, 36(6): 649-654. (in Chinese)

[10] RODI W. Comparison of LES and RANS calculations of the flow around bluff bodies[J]. Journal of Wind Engineering and Industrial Aerodynamics, 1997, 69-71: 55-75.

[11] ZHAO Wei, Huhe Aode. Experimental investigation of scour mechanism around a circular cylinder[J]. Acta Mechanica Sinica, 2006, 38(5): 577-585. (in Chinese)

[12] YUHI M., ISHIDA H., UMEDA. S. A numerical study of three-dimensional flow fields around a vertical cylinder mounted on a bed[J]. Coastal Structures, 1999, 18(4): 783-792. 